young type 1 diabetic patients. Pediatr Endocrinol Rev. 2008;5 (suppl 4):958-963.

35. Shao JS, Cheng SL, Sadhu J, Towler DA. Inflammation and the osteogenic regulation of vascular calcification: a review and perspective. Hypertension. 2010;55(3):579-592.

36. Zhao MM, et al. Mitochondrial reactive oxygen species promote $\mathrm{p} 65$ nuclear translocation mediating high-phosphate-induced vascular calcification in vitro and in vivo. Kidney Int. 2011;79(10):1071-1079.

37. Wang $S$, et al. AMPKalpha2 deletion causes aberrant expression and activation of $\mathrm{NAD}(\mathrm{P}) \mathrm{H}$ oxidase and consequent endothelial dysfunction in vivo: role of 26S proteasomes. Circ Res. 2010;106(6):1117-1128.

38. Wang S, et al. Activation of AMP-activated protein kinase alpha2 by nicotine instigates formation of abdominal aortic aneurysms in mice in vivo. Nat Med. 2012;18(6):902-910.

39. Dasgupta $B$, et al. The AMPK $\beta 2$ subunit is required for energy homeostasis during metabolic stress. Mol Cell Biol. 2012;32(14):2837-2848.

40. Bogachus LD, Turcotte LP. Genetic downregulation of AMPK-alpha-isoforms uncovers the mechanism by which metformin decreases FA uptake and oxidation in skeletal muscle cells. Am J Physiol Cell Physiol. 2010;299(6):C1549-C1561.

41. Rhee EP, et al. A combined epidemiologic and metabolomic approach improves CKD prediction. J Am Soc Nephrol. 2013;24(8):1330-1338.

42. Kiebish MA, et al. Dynamic simulation of cardiolipin remodeling: greasing the wheels for an interpretative approach to lipidomics. J Lipid Res. 2010;51(8):2153-2170.

\title{
Regulatory T cells use "Itch" to control asthma
}

\author{
WanJun Chen
}

Mucosal Immunology Section, OPCB, NIDCR, NIH, Bethesda, Maryland, USA.

\begin{abstract}
Regulatory $\mathrm{T}$ cells (Tregs) control type $2 \mathrm{~T}$ helper cell-mediated (Th2-mediated) lung inflammation, but the molecular mechanisms by which Tregs execute this activity remain elusive. In this issue of the JCI, Jin et al. reveal that Itch, a HECT-type E3 ubiquitin ligase in Tregs, plays a specific role in restraining Th2 cell responses. This finding has important implications for understanding the pathogenesis of allergy and asthma.
\end{abstract}

\section{Introduction}

Tregs, which are characterized by the expression of the transcription factor Foxp3, are instrumental in the induction and maintenance of immune tolerance and homeostasis $(1,2)$; however, the molecular mechanisms underlying Treg-mediated immunoregulatory functions remain elusive. This issue is complex, considering that Tregs are capable of executing their immunosuppressive activity against a broad and diverse array of antigens and within different microenvironments. For example, Tregs can suppress IFN- $\gamma$-producing Th1, IL-17-producing Th17, and IL-4-producing Th2 responses. This leads to the question: Do Tregs use universal suppressive mechanisms or do these cells employ environmentally orientated programs of suppression enacted in response to distinct inflammatory cues?

It is generally accepted that Tregs use an arsenal of mechanisms to suppress the immune response through certain surface molecules (e.g., CTLA4, CD25, CD73, CD39) and secretion of immunoregulatory cytokines (e.g., TGF- $\beta$, IL-10) $(3,4)$. These mechanisms explain many, but not all, of the immunosuppressive activities of Tregs. Recent evidence suggests that

Conflict of interest: The author has declared that no conflict of interest exists.

Citation for this article: J Clin Invest. 2013; 123(11):4576-4578. doi:10.1172/JCI72477.
Tregs suppress different types of T cellmediated immune responses through the acquisition of specific $\mathrm{T}$ effector cell transcriptional programs, depending on the context and the location of inflammation (5). For example, Treg-mediated specific suppression of Th1 cells requires the expression of the transcription factor T-box 21 (TBET). Treg-specific deletion of Tbet results in uncontrolled type 1 inflammation (6). In a similar vein, Treg-specific deletion of the gene encoding STAT3 leads to dysregulated Th17 responses (7), implying a key role for STAT3 in Treg control of Th17-mediated inflammation. Intriguingly, Treg-specific knockout of Irf4, a transcription factor involved in both Th2 and Th17 cell differentiation, causes the selective dysregulation of autoreactive Th2 responses, suggesting that IRF4 is required for Treg suppression of Th2 cells (8). In this issue of the JCI, Jin et al. (9) reveal an indispensable function of Itch, a HECT (homologous to E6-associated protein C terminus) E3 ubiquitin ligase, in Treg-regulated Th2 responses in mice. Targeted deletion of Itch in Foxp $3^{+}$cells resulted in the uncontrolled production of IL-4, IL-5, and IL-13 by Tregs and, surprisingly, by Itch-sufficient $\mathrm{CD}^{+}$effector T cells (9).

\section{Without Itch in Tregs, Th2-type inflammation is uncontrolled}

Itch was originally identified in a mutant mouse that displayed skin scratching and abnormal immune disorders (10). $\mathrm{Itch}^{-/-}$mice exhibit swollen lymph nodes, enlarged spleens, and increased Th2-type inflammation in the lungs and digestive tract (11). The excess Th2 inflammation in these mice was attributed to the inability of $\mathrm{Itch}^{-/-} \mathrm{CD} 4^{+} \mathrm{T}$ cells to differentiate into inducible Tregs (iTregs ) $(12,13)$ in response to TGF- $\beta$ (14); however, an intrinsic role for Itch in thymic-derived Treg cells ( $\mathrm{t}$ Tregs or nTregs) remains unknown.

Jin et al. developed a Treg-specific Itch knockout mouse by crossing Itchf/f mice with Foxp $3^{\mathrm{Cre}}$ mice (Itchf/fFoxp $3^{\mathrm{Cre}}$ ) to investigate the role of Itch in tTregs. Surprisingly, the Itchf/fFoxp $3^{\text {Cre }}$ mice appeared normal at birth, but later exhibited lymphoproliferative disorder, pulmonary inflammation, skin lesions, decreased weight, and a higher mortality rate. Since Itch regulates Th2 cytokine production (11), the authors challenged Itchf/fFoxp $3^{\mathrm{Cre}}$ mice with OVA in an experimental model of asthma. They found that compared with control mice, Itchf/fFoxp $3^{\text {Cre }}$ mice had more severe lung inflammation with dramatic increases in OVA-specific IgE and Th2 cytokines including IL-4, IL-5, and IL-13 in the BAL. These results raised the possibility that aberrant Th2 inflammation was a systemic event in Itchf/fFoxp $3^{\mathrm{Cre}}$ mice.

To address the possibility that Th2 inflammation is systemically altered in Itchf/fFoxp $3^{\text {Cre }}$ mice, Jin et al. examined animals between 6 and 8 weeks of age, when signs of inflammation first appeared. There were no changes in the thymus, but the number of splenic $\mathrm{CD}^{+}$and $\mathrm{CD}^{+} \mathrm{T}$ cells was increased along with activated $\mathrm{T}$ cells in Itchf/fFoxp $3^{\mathrm{Cre}}$ mice compared with age-matched WT mice. Importantly, ex vivo analysis revealed that $\mathrm{CD}^{+} \mathrm{T}$ cells 
A
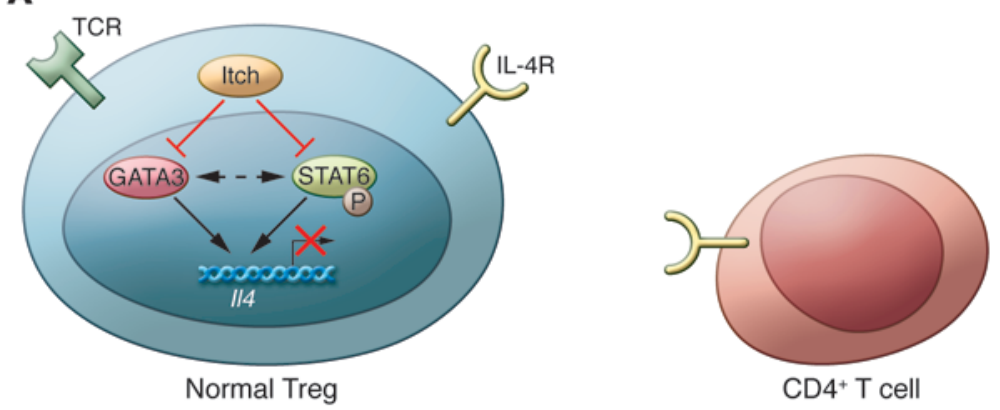

B

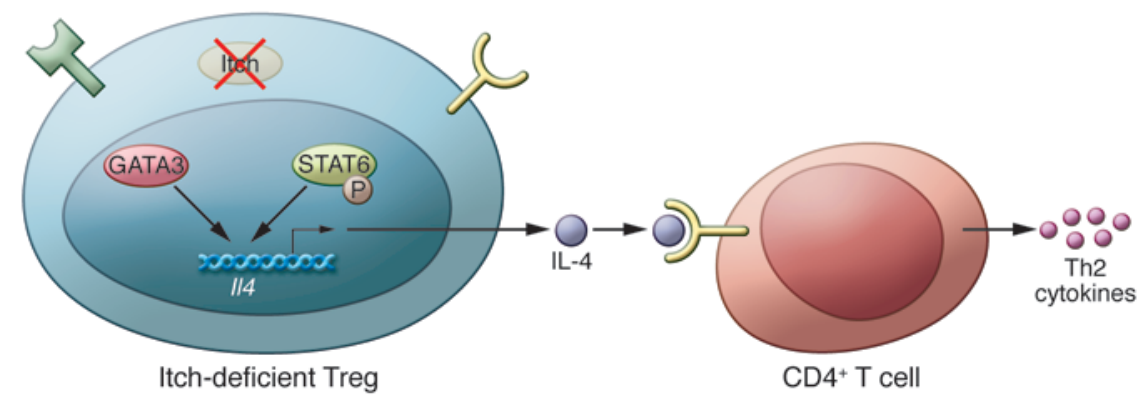

\section{Figure 1}

Itch regulates Th2 cytokines in Tregs. (A) In normal Tregs, Itch controls the expression of GATA3 and phosphorylation of STAT6, which prevents //4 gene transcription and IL-4 production. Thus, no Th2 cytokines (IL-4, IL-5, and IL-13) are produced or secreted by Itch-sufficient normal T cells; (B) In the absence of Itch, as evident in Itch ${ }^{\text {ft/ }}$ Foxp $^{\text {Cre }}$ mice, Tregs cannot control GATA3 and STAT6, which results in increased GATA3 expression and STAT6 activation. Consequently, I/4 transcription is activated and IL-4 production is increased. IL-4 secreted by Itch-deficient Tregs instructs the normal naive CD4+ $T$ cells to initiate a Th2 cell differentiation program for the secretion of large amounts of IL-4, IL-5, and IL-13, which together lead to uncontrolled Th2-type inflammation, especially in the lungs of Itch ${ }^{f / f} F o x p 3^{C r e}$ mice. Red "X" indicates a block or deletion of the target molecules; black arrows indicate positive effects, and red lines indicate negative effects. Dotted line indicates no available evidence yet. from the spleen and peripheral lymph nodes produced larger amounts of the Th2 cytokines IL-4, IL-5, and IL-13, but normal levels of Th1 and Th17 cytokines; however, $\mathrm{CD}^{+} \mathrm{T}$ cells isolated from the lungs of Itchf/fFoxp $3^{\mathrm{Cre}}$ mice had a higher proportion of Th17 cells along with increased Th2 cytokines. These findings suggest that a lack of Itch in Tregs leads to systemic $T$ cell activation, which skews toward Th2 cytokine production.

It is known that a reduction in Treg numbers, downregulation of Foxp3 expression, and a defective suppressive ability and/or switch of Treg phenotype by loss of Foxp3 (ex-Tregs) all contribute to Treg dysfunction. Jin et al. investigated whether the deletion of Itch in Tregs affects their homeostasis, suppressive function, or stability. They found that Treg-specific deletion of Itch did not change Treg numbers in the thymus or periphery. Standard in vitro coculture suppression assays (4) and an in vivo $\mathrm{CD}^{+}{ }^{+} \mathrm{CD} 45 \mathrm{RB}^{\text {hi }} \mathrm{T}$ cell transfermediated colitis model (15) revealed normal suppressive function of Itch-deficient Tregs. Furthermore, Itch knockout Tregs exhibited normal levels of Foxp3 and had a similar frequency of conversion to Foxp3negative "ex-Tregs" in the absence or presence of TCR stimulation. Thus, Itch did not control the Foxp3 expression, suppressive function, or stability of Tregs. In which case, what are the exact mechanisms responsible for aberrant Th2 cytokine production in Itchf/fFoxp $3^{\mathrm{Cre}}$ mice?

\section{Itch-deficient Tregs have Th2 properties}

An examination of cytokine production by Itch-deficient Tregs revealed that they produced large amounts of Th2 cytokines in vitro. Furthermore, Itch-deficient, Foxp3negative "ex-Tregs" produced even greater amounts of Th2 cytokines. The unrestrained Th2 cytokine production in Itch-deficient Tregs was accompanied by $\mathrm{T}$ cell activation and increased expression of some Treg-associated markers; however, it is not clear whether changes in Treg markers are important in the immune response. Itch-deficient Tregs also produced enhanced Th2 cytokines in an OVAmediated experimental model of allergic asthma. Together, these findings reveal a previously unrecognized function of Itch in preventing the acquisition of Th2-like properties in Tregs.

Examination of the expression of transcription factors critical for driving the Th2 differentiation program revealed that expression of the GATA-binding protein 3 gene (Gata3) and the transcription factor cMaf were increased in Itch-deficient Tregs. TCR stimulation further increased GATA3 expression and promoted phosphorylation of STAT6 in Itch-deficient Tregs. Importantly, the functional relevance of GATA3 and STAT6 in promoting Th2 cytokines in Itch-deficient Tregs was confirmed by Gata 3 and Stat 6 knockdown experiments. The reduction of both Gata3 and Stat6 expression inhibited Th2 cytokine production by Itch-deficient Tregs. These findings indicate that Itch controls the activities of GATA3 and STAT6 and thus restrains Tregs from acquiring the ability to produce Th2 cytokines.

\section{Itch-deficient Tregs instruct $T$ cells to produce IL-4}

Despite the dramatic Th2 cytokine production by Itch-deficient Tregs, these cells constitute only a small portion of total $\mathrm{T}$ cells. Jin et al. reasoned that the Th2 cytokines produced by Itch-deficient Tregs could not fully account for the pathogenic Th2 inflammatory response seen in $I t c h f / f F o x p 3^{C r e}$ mice. They hypothesized that the cytokines produced by Itch-deficient Tregs instruct naive normal $\mathrm{T}$ cells to develop into Th2 cells. Indeed, they found that naive $\mathrm{CD} 4^{+} \mathrm{T}$ cells cocultured with supernatants derived from TCR-stimulated Itch-deficient Tregs differentiated into IL-4- and IL-5-producing $\mathrm{T}$ effector cells. Treatment with an anti-IL-4 antibody completely abolished Th2 differentiation, indicating that IL-4 production by Itch-deficient Tregs instructs the development of a Th2 inflammatory response. 


\section{Summary and perspectives}

Itch has been shown to be a unique "player" on a team of newly emerging molecules that are capable of regulating Treg function. It appears that the major task Itch performs is to restrain Tregs from producing Th2 cytokines. This is largely through the suppression of Gata3 expression and STAT6 phosphorylation. In the absence of this checkpoint, Itch-deficient Tregs produce IL-4, which drives nonTregs to the Th2 phenotype (Figure 1). The function performed by Itch in Tregs is therefore different from that of other factors shown to be vital for Treg suppressive activities. In Tregs, IRF4 forms a complex with Foxp 3 that confers the ability to control Th2 responses (8); however, GATA3 expression in Tregs is vital to maintaining Treg suppressive function and stability $(16,17)$. The Itch/GATA3 pathway presented in this article may represent a specific part of the broad functional spectrum of GATA3 in Tregs. It will be important to elucidate the molecular pathways by which Itch selectively inhibits GATA3mediated Th2 responses. In addition to uncontrolled Th2 inflammation, it is surprising that Itch deficiency did not change the frequency at which Tregs converted to Foxp3-negative "ex-Tregs," and this finding supports the case for Treg "plasticity" in response to inflammatory cues.

The elucidation of the requirement for Itch in Tregs to control Th2 inflammation raises several exciting and intriguing questions. First, what is the role of iTregs in the uncontrolled Th2 lung inflammation induced by Itch deletion specifically in tTregs? It is known that iTregs are critical for suppressing Th2 responses in the lung $(12,14,18)$, and $\mathrm{CD}^{+} \mathrm{T}$ cells from systemic $\mathrm{Itch}^{-/-}$mice exhibit a severe defect in iTreg conversion. Second, what are the underlying mechanisms responsible for the increased activation and proliferation of both Itch-deficient Tregs and Itch-sufficient non-Treg cells in Itchf/fFoxp $3^{\text {Cre }}$ mice? Does Itch function via Foxp3-independent mechanisms to control $\mathrm{T}$ cell activation? Third, why are Th17 cells enhanced in the lung in experimental asthma, but not in the CNS during EAE in Itchf/fFoxp 3 Cre mice? Finally, what is/are the molecular mechanism(s) by which Itch regulates GATA3 and STAT6? Does phosphorylation of STAT6 act on GATA3-dependant gene expression or vice versa during the enhanced IL-4 production seen with Itch deficiency? Although it was reported that Itch regulates JunB-mediated Il4 gene transcription in normal $\mathrm{T}$ cells, Tregs do not express JunB at steady state or when activated. The authors consider that Itch may regulate the epigenetic codes present at the genes encoding Th2 cytokines. Whatever proves true, this report by Jin et al. clearly reveals that Tregs use "Itch" to specifically control Th2 inflammatory responses and may pave the way for a deeper understanding of the pathogenesis of human allergy and asthma.

\section{Acknowledgments}

I thank Joanne E. Konkel, Cheryl Chia, and Eric Tu of the Mucosal Immunology Section, NIDCR, NIH for critically reading the manuscript. This work was supported by the Intramural Research Program of the NIH, NIDCR.

Address correspondence to: WanJun Chen, Mucosal Immunology Section, NIDCR, NIH, 30 Convent Dr., Bethesda, Maryland 20892, USA. Phone: 301.435.7168; Fax: 301. 402.1064; E-mail:wchen@dir.nidcr.nih.gov.

\footnotetext{
1. Littman DR, Rudensky AY. Th17 and regulatory T cells in mediating and restraining inflammation. Cell. 2010;140(6):845-858.

2. Ohkura N, Kitagawa Y, Sakaguchi S. Development
}

and maintenance of regulatory $\mathrm{T}$ cells. Immunity. 2013;38(3):414-423.

3. Chen W, Wahl SM. TGF- $\beta$ : the missing link in $\mathrm{CD} 4+\mathrm{CD} 25+$ regulatory $\mathrm{T}$ cell-mediated immunosuppression. Cytokine Growth Factor Rev. 2003; 14(2):85-89.

4. Shevach EM. Mechanisms of foxp $3^{+} \mathrm{T}$ regulatory cell-mediated suppression. Immunity. 2009; 30(5):636-645.

5. Chaudhry A, Rudensky AY. Control of inflammation by integration of environmental cues by regulatory T cells. J Clin Invest. 2013;123(3):939-944.

6. Koch MA, Tucker-Heard G, Perdue NR, Killebrew JR, Urdahl KB, Campbell DJ. The transcription factor T-bet controls regulatory $\mathrm{T}$ cell homeostasis and function during type 1 inflammation. Nat Immunol. 2009;10(6):595-602.

7. Chaudhry A, et al. CD4 ${ }^{+}$regulatory $\mathrm{T}$ cells control TH17 responses in a Stat3-dependent manner. Science. 2009;326(5955):986-991.

8. Zheng Y, et al. Regulatory T-cell suppressor program co-opts transcription factor IRF4 to control T(H)2 responses. Nature. 2009;458(7236):351-356.

9. Jin H, Park Y, Elly C, Liu Y-C. Itch expression by Treg cells controls Th2 inflammatory responses. J Clin Invest. 2013;123(11):4923-4934.

10. Perry WL, Hustad CM, Swing DA, O'Sullivan TN, Jenkins NA, Copeland NG. The itchy locus encodes a novel ubiquitin protein ligase that is disrupted in a18H mice. Nat Genet. 1998;18(2):143-146.

11. Liu YC. The E3 ubiquitin ligase Itch in T cell activation, differentiation, and tolerance. Semin Immunol. 2007;19(3):197-205.

12. Fang $D$, et al. Dysregulation of T lymphocyte function in itchy mice: a role for Itch in TH2 differentiation. Nat Immunol. 2002;3(3):281-287.

13. Venuprasad K, et al. The E3 ubiquitin ligase Itch regulates expression of transcription factor Foxp3 and airway inflammation by enhancing the function of transcription factor TIEG1. Nat Immunol. 2008;9(3):245-253.

14. Chen $\mathrm{W}$, et al. Conversion of peripheral $\mathrm{CD}^{+}$ $\mathrm{CD} 25^{-}$naive $\mathrm{T}$ cells to $\mathrm{CD} 4^{+} \mathrm{CD} 25^{+}$regulatory $\mathrm{T}$ cells by TGF- $\beta$ induction of transcription factor Foxp3. J Exp Med. 2003;198(12):1875-1886.

15. Powrie F, Correa-Oliveira R, Mauze S, Coffman RL. Regulatory interactions between CD45RBhigh and CD45RBlow $\mathrm{CD}^{+} \mathrm{T}$ cells are important for the balance between protective and pathogenic cell-mediated immunity. J Exp Med. 1994;179(2):589-600.

16. Wang Y, Su MA, Wan YY. An essential role of the transcription factor GATA-3 for the function of regulatory T cells. Immunity. 2011;35(3):337-348.

17. Wohlfert EA, et al. GATA3 controls Foxp $3^{+}$regulatory $\mathrm{T}$ cell fate during inflammation in mice. J Clin Invest. 2011;121(11):4503-4515.

18. Josefowicz SZ, et al. Extrathymically generated regulatory $\mathrm{T}$ cells control mucosal TH2 inflammation. Nature. 2012;482(7385):395-399. 